\title{
Prediction of peak plantar pressure for diabetic foot: the regressional model.
}

HAZARI, A., MAIYA, A., AGOURIS, I., MONTEIRO, A., SHIVASHANKARA. 


\section{RESEARCH PAPER HIGHLIGHTS}

KNOWN FACTS- Diabetes Mellitus is a potential epidemic in Asia and India being the diabetic capital. Diabetes causes various foot complications with underlying peripheral neuropathy. The most important etiological factor for a diabetic foot is high/altered plantar pressure.

\section{Novelty and Highlights:}

1. The study determined the peak plantar pressure difference between type 2 diabetes mellitus with and without neuropathy

2. The study focused on the most important clinical parameters that could be associated with peak plantar pressure in type 2 diabetes mellitus participants.

3. The clinical variables like severity of neuropathy, varicosity, plantar cushioning, dynamic knee joint angle, and angular ankle joint velocity were important predictors for peak plantar pressure. 
Title: A Clinical Tool For Diabetic Foot Prediction: The Regressional Model

Authors and Affiliations: Dr. Animesh Hazari ${ }^{~}$, Dr Arun Maiya ${ }^{2 *}$, Dr Ioannis Agouris ${ }^{3}$, Ashma Monteiro ${ }^{4}$, Dr Shivashankara ${ }^{5}$

1. Dr. Animesh Hazari

$\mathrm{PhD}$ Scholar,

Department of Physiotherapy,

School of Allied Health Sciences,

Manipal Academy of Higher Education, Karnataka, India-576104.

Email Id:animeshh8@gmail.com

2. Dr. Arun G Maiya PT,PhD

Professor and Associate Dean,

Department of Physiotherapy,

School of Allied Health Sciences,

Manipal Academy of Higher Education, Karnataka, India-576104.

Email Id: arun.maiya.g@gmail.com, ajmaiya@gmail.com

3. Dr.Ioannis Agouris, BSc, PgDip, PhD

Professor, Robert Gordon University

Garthdee House, Garthdee Road, Aberdeen AB10 7AQ, United Kingdom

Email Id: i.agouris@rgu.ac.uk

4. Ashma Monteiro

Assistant Professor,

Prasanna School of Statistics, Department of Bio-statistics,

Manipal Academy of Higher Education Udupi, Karnataka, India- 576104

Email id: ashma.monteiro@ manipal.edu 
5. Dr.Shivashankara KN MD, MBBS

Professor,

Department of General Medicine,

Kasturba Medical College,

Manipal Academy of Higher Education Udupi, Karnataka, India- 576104

Email Id: $\underline{\text { shi.sha@manipal.edu }}$

\section{Corresponding author:}

Dr. Arun G Maiya, Professor and Associate Dean, Department of Physiotherapy,

School of Allied Health Sciences, Manipal Academy of Higher Education,

Karnataka, India-576104.

Email Id: arun.maiya.g@gmail.com, ajmaiya@gmail.com; Ph.: +919845350823 
1 Prediction of Peak Plantar Pressure for Diabetic Foot: The Regressional

2 Model

3 Abstract:

Background: The increase in peak plantar pressure could be the most important etiological factor for pathogenesis of a Diabetic Foot. Thus the fate of a diabetic foot syndrome which is a clinical triad of neurological, vascular and musculoskeletal changes could be biomechanically predictive and preventive using clinical parameters. In the presence of peripheral neuropathy, certain clinical parameters could be severely altered resulting into increased peak plantar pressure. Therefore the aim of the study was to identify the most important clinical parameters for the prediction of peak plantar pressure between neuropathy and non-neuropathy type 2 diabetes mellitus participants. Methodology: A total of 380 participants were recruited under the study and divided into two groups (190 each group). The cross-sectional study was conducted at Kasturba Hosipal, Manipal,India. Multiple regression analysis was performed to find the hyperplane of best fit. Stepwise regression was performed witha( entry $=.15$ and $\alpha$ removal= .2) to select the best subset of predictors. Results: Adjusted R2 of the final model which included the predictors showed $90.8 \%$ variability for the dependent variable. Conclusion: The findings from the regression analysis and suggested model was found be strongly significant in predicting the peak plantar pressure between neuropathy and non-neuropathy type 2 diabetes mellitus participants. Since higher values of peak plantar pressure is strongly associated with risk for future diabetic foot complications, it could be suggested that these clinical parameters could be very useful to assess and should be used in routine clinical practice very effectively. 
INTRODUCTION: Foot complications are the most ignored part of diabetes management. The increase in peak plantar pressure could be the most important etiological factor for pathogenesis of a Diabetic Foot [2]. Diabetic Foot Syndrome (DFS) as defined by the World Health Organization is an "ulceration of the foot (distally from the ankle and including the ankle) associated with neuropathy and different grades of ischemia and infection" [3]. Thus the fate and pathophysiology of a diabetic foot syndrome include a clinical triad of neurological, vascular and musculoskeletal changes which could be biomechanically predictive and preventive using clinical parameters. In the presence of peripheral neuropathy, certain clinical parameters could be severely altered resulting into increased peak plantar pressure [1]. For e.g. the sensory deficit leads to common foot complications like altered sensations (tingling,burning, pricking, hypoesthesia, allodynia). The sensory deficit could be clinicaly manifested with the loss of protective sensation initially (touch and temperature), and progression to damage of large diameter sensory fibers (vibration loss) [4]. The motor neuropathy presents as weakness and atrophy of intrinsic and extrinsic foot muscles at ankle, and leads to common foot deformities like claw toes, hammer toes, equinus, Charcot foot, changes in foot arch, tightness of plantar aponeurosis, etc. The primary changes in the musculoskeletal structures could also be associated with consequent secondary changes in joint structure and function like the decreased range of motion [5]. The vascular changes are often seen as reduced blood supply to peripheral microvasculature of foot. Vascular insufficiency may be clinically manifested by the altered ankle brachial index (ABI), blackish discoloration of the foot, altered temperature of the foot. Autonomic neuropathy and dermatological changes are the most common manifestation that accounts for 47.5-91.2\% of people with type 2 diabetes mellitus [6]. Decreased blood circulation can lead to changes in the skin collagen altering its texture, appearance, and ability to heal. As a result, the skin's Diabetes peripheral neuropathy (DPN), Diabetic Foot Syndrome (DFS), Ankle Brachial Index (ABI), Ground Reaction Force (GRF), Body Mass Index (BMI), 
endothelial cells get damaged, and this may even reduce its ability to sweat which leads to dry skin, fissure and callus formation as well as a decrease in the ability to sense temperature and pressure [7].Studies have reported that the increase in peak plantar pressure and repetitive micro trauma due to higher ground reaction force (GRF) could be the most important etiological factor for pathogenesis of a DFS [8].Similar finding were supported by another study which concluded 57\% higher risk for ulceration at high pressure points. The individual areas of foot like hallux, metatarsal heads, midfoot and heel were positively associated with the peak plantar pressure and incidence of foot ulcers [9]. While studying the presentation and causative factors, it is now understood that DFS could be biomechanically determinative predominately by increased plantar pressure/ ground reaction force. Thus with a detailed clinical evaluation, and identification of clinical parameters which could be closely associated with high plantar pressure, the risk of future diabetic foot complications could be predicted and prevented. Therefore the aim of the study was to identify the most important clinical parameters of diabetic foot which could be the predictors of the peak plantar pressure in diabetic foot. The objectives of the study were as follows:

1.To find a line of hyper plane between the neurological, vascular, musculoskeletal and biomechanical findings with maximum/peak plantar pressure among participants with type 2 diabetes mellitus.

2. To provide regression equation and prediction model for peak plantar pressure distribution between neuropathy and non-neuropathy type 2 diabetes mellitus participants.

\section{METHODOLOGY:}

Diabetes peripheral neuropathy (DPN), Diabetic Foot Syndrome (DFS), Ankle Brachial Index (ABI), Ground Reaction Force (GRF), Body Mass Index (BMI), 
Study Design and Settings: The observational cross-sectional study was conducted at Diabetic Foot Clinic, Kasturba Hospital, Manipal, Karnataka India. The study is a part of $\mathrm{PhD}$.

Study Population: All diagnosed type 2 diabetes mellitus participants were recruited under the purposive sampling method. A total of 380 participants (the sample size was taken as a part of $\mathrm{PhD}$ study using the formula for comparison of mean for outcome like peak plantar pressure) were recruited under the study. The participants were divided into two equal groups $(n=190$ each) in order to determine the change in plantar pressure distribution and its association with clinical parameters between participants with type 2 DM with neuropathy and type 2 DM without neuropathy . Neuropathy subjects have been graded and compared with non-neuropathy group as the reference in the equation

Study Procedure: The ethical clearance for was obtained from Institutional Ethics Committee. An informed consent was obtained from all participants following which a detailed diabetic foot evaluation was taken including neurological, vascular, musculoskeletal and biomechanical findings. It is well known that plantar pressure is severely affected by presence of peripheral neuropathy. Therefore screening for the presence of diabetes peripheral neuropathy is important. The presence of neuropathy was confirmed with findings from Monofilament and Vibration pressure threshold (VPT) values. The VPT values were also used to further stratify the grades of neuropathy. A value of 1 to 14 volts was reported as the absence of neuropathy, 14 to 20 volts as a risk for neuropathy, and values above 20 volts were considered as neuropathy among Indian population based on previous literature [10]. The protective sensation testing was performed using the standard procedure for 5.07/10g Semmes Weinstein Monofilament Test and vibration sense testing using biothesiometer (Vibration Pressure threshold Diabetes peripheral neuropathy (DPN), Diabetic Foot Syndrome (DFS), Ankle Brachial Index (ABI), Ground Reaction Force (GRF), Body Mass Index (BMI), 
Device/VPT). Semmes Weinstein $10 \mathrm{~g}$ Monofilament Testing is a widely used neuropathy screening tool in diabetes mellitus. A systematic review was done on the use of Semmes Weinstein Monofilament as a diabetic neuropathy screening tool used in various studies. The study concluded that it had a sensitivity ranging from $57 \%$ to $93 \%$, specificity ranging from $75 \%$ to $100 \%$ and a positive predictive value ranging from $84 \%$ to $100 \%$ whereas negative predictive value is ranging from $36 \%$ to $94 \%$ [11]. Similar to $10 \mathrm{~g}$ Monofilament, VPT testing is a valid, reliable and cost-effective clinic tool to diagnose neuropathy compared to a gold standard like Nerve Conduction Velocity. A study was conducted by Kaur and Singh (2016) to test VPT as a measure of distal symmetrical polyneuropathy (DSPN) in type 2 diabetes mellitus [12]. The study concluded that VPT was a reliable measure for DSPN with good sensitivity (74.07\%) and specificity to diagnose clinical neuropathy. Following the confirmation of DPN, other clinical variables were obtained which consisted of more than 150 direct variables and 348 sub-variables into the assessment. The variables includes in the study were selected and categorized based on the standard diabetic foot evaluation and their association with maximum plantar pressure. In the present study we have listed the variables that could alter the plantar pressure directly or indirectly as listed here. The independent variables (continuous and categorical) consisted of group, gender,age, height, weight, body mass index (BMI), duration of diabetes, occupation, Fasting blood sugar (FBS), post-prandial blood sugar (PPBS), HbA1c, ankle brachial index (ABI), type of hypoglycemic agent (oral, insulin etc),smoking, alcohol, family history, ankle static angle (ASA), ankle heel-strike angle (AHSA), ankle toe-off angle (ATOA), knee static angle (KSA), knee heelstrike angle (KHSA), knee mid-stance angle (KMSA), knee toe-off angle (KTOA), ankle heel-strike velocity (AHSV), ankle mid-stance velocity (AMSV), ankle toeoff velocity (ATOV), knee heel-strike velocity (KHSV), knee mid-stance velocity Diabetes peripheral neuropathy (DPN), Diabetic Foot Syndrome (DFS), Ankle Brachial Index (ABI), Ground Reaction Force (GRF), Body Mass Index (BMI), 
(KMSV), knee toe-off velocity (KTOV), ankle heel-strike acceleration (AHSAA), ankle midstance acceleration (AMSAA), ankle toe-off acceleration (ATOAA),knee heel-strike acceleration (KHSAA), knee mid-stance acceleration (KMSAA), knee toe-off acceleration (KTOAA), sensation (touch), ankle reflex, pedal pulse, $10 \mathrm{~g}$ monofilament testing, grades of neuropathy (VPT), muscle tightness, dryness of skin, discoloration of feet, toe deformities like hallux valgus, clawing and hammer toes, ingrown nails, callus, fissures, prominent metatarsal heads, peripheral vascular disease, obesity, hypertension, levels of physical activity, vascular and neurological claudication, pedaledema, varicosity, nephropathy ,retinopathy plantar fasciitis ,flexible footwear, rigid footwear ,specialised footwear (micrcellulose rubber), plantar fat pad, max.pressure area, step-time, swing time, double-stance time, gait cycle time, stride time, step-length, gait cycle length, foot angle, foot archtype (cavus/planus), first ray length, fifth ray length, Naviculardrop height, foot posture index (FPI), extensor halluces strength (EHMMT), ankle dorsiflexor strength (ADMMT), plantar flexor strength (PFMMT), knee flexor strength (KFMMT), hip abductor strength (HABMMT), gastrocnemius tightness , soleus tightness, quadriceps tightness, hip adductor tightness, hip abductor tightness, Illio-tibial band tightness, hamstring tightness, Q angle, Neuropathy scales including Michigan Neuropathy Screening Instrument, Leeds Asseesment of Neuropathy Signs and Symptoms, Douleur Neuropathique 4, Neuropathy disability score, Neuropathy Symptoms score respectively (MNSIA, MNSIB, LANSS, DN4, NDS, NSS), postural analysis including forward neck, forward shoulder, cervicallordosis, kyphosis, lumbar lordosis, scoliosis, pelvis tilt, femoral rotation, genu valgus/varum, patella shift, tibia torsion, and calcaneum neutral. Various subanalysis were also perform and variables were used. Since it is well known that higher plantar pressure is the most important outcome for predicting diabetic foot 
syndrome and future ulcerations, we selected peak/maximum pressure as the 163 dependent variable.

164 The biochemistry profile for all diabetes mellitus participants were taken from the laboratory findings. The clinical assessment for detection of peripheral neuropathy was performed as per the standard guidelines using MNSI $10 \mathrm{~g}$ Monofilament, and VPT testing by a clinician. The muscle strength was determined by the Manual Muscle Testing Grading system by a skilled physiotherapist. For kinematic analysis 2D/3D SIMI Motion GmbH analysis system was used using two high speed infrared cameras and $9 \mathrm{~mm}$ retro-reflective marker sets for ankle and knee joints [23]. Motion analysis system is the gold standard tool for determining the joint kinematics in the current state of art. The kinetic analysis for peak plantar pressure was obtained using the Wintrack Dyanamic Foot Scanner (MEDICAPTEURS Technology France). The data was captured for barefoot analysis.

Statistical Analysis: The data was analyzed using SPSS15. Multiple regression analysis was performed to find the hyperplane of best fit. Stepwise regression was performed with $\left(\alpha_{\text {entry }}=.15\right.$ and $\left.\alpha_{\text {removal }}=.2\right)$ to select the best subset of predictors. The predictors in the final model had Variance Inflation Factor less than 5 which confirms the absence of possible multicollinearity between predictors. Comparison of neuropathy subjects at various grades with non-neuropathy subjects as reference have been performed by Wald $t$ test and reported in the table with $\mathrm{p}$ values.

RESULTS: The multiple linear regression analysis for prediction of maximum plantar pressure was performed. The descriptive data for blood profile and anthropometry has been shown in Table 1 below. Table 2 represents the duration of diabetes mellitus and severity grading among the neuropathy group of 
participants. Table 3 represents the estimation of maximum plantar pressure using

188 the regression model.

189 Table 1: Descriptive for Anthropometric and Blood profile of all Participants

\begin{tabular}{|c|c|c|c|}
\hline VARIABLES & GROUP & GENDER & MEAN $\pm S . D$ \\
\hline \multirow[b]{2}{*}{ AGE in years } & $\begin{array}{l}\text { NEUROPATHY } \\
(\mathrm{n}=190)\end{array}$ & $\begin{array}{l}\text { OVERALL } \\
\text { MALE (145) } \\
\text { FEMALE (45) }\end{array}$ & $\begin{array}{l}57.65 \pm 10.77 \\
58.98 \pm 10.56 \\
50.60 \pm 10.17\end{array}$ \\
\hline & $\begin{array}{l}\text { NON-NEUROPATHY } \\
(n=190)\end{array}$ & $\begin{array}{l}\text { OVERALL } \\
\text { MALE (142) } \\
\text { FEMALE (48) }\end{array}$ & $\begin{array}{l}53.12 \pm 10.36 \\
53.65 \pm 10.24 \\
50.51 \pm 10.61\end{array}$ \\
\hline \multirow{2}{*}{ HEIGHT in $\mathrm{cm}$} & $\begin{array}{l}\text { NEUROPATHY } \\
(\mathrm{n}=190)\end{array}$ & $\begin{array}{l}\text { OVERALL } \\
\text { MALE (145) } \\
\text { FEMALE (45) }\end{array}$ & $\begin{array}{l}164.57 \pm 8.22 \\
167.36 \pm 6.13 \\
152.5 \pm 4.44\end{array}$ \\
\hline & $\begin{array}{l}\text { NON-NEUROPATHY } \\
(n=190)\end{array}$ & $\begin{array}{l}\text { OVERALL } \\
\text { MALE (142) } \\
\text { FEMALE (48) }\end{array}$ & $\begin{array}{l}164.98 \pm 8.55 \\
167.4 \pm 6.97 \\
154.19 \pm 5.94\end{array}$ \\
\hline \multirow[t]{2}{*}{ WEIGHT in $\mathrm{kg}$} & $\begin{array}{l}\text { NEUROPATHY } \\
(n=190)\end{array}$ & $\begin{array}{l}\text { OVERALL } \\
\text { MALE (145) } \\
\text { FEMALE (45) }\end{array}$ & $\begin{array}{l}71.26 \pm 10.62 \\
72.66 \pm 10.4 \\
65.16 \pm 9.63\end{array}$ \\
\hline & $\begin{array}{l}\text { NON-NEUROPATHY } \\
(n=190)\end{array}$ & $\begin{array}{l}\text { OVERALL } \\
\text { MALE (142) } \\
\text { FEMALE (48) }\end{array}$ & $\begin{array}{l}70.32 \pm 10.22 \\
71.09 \pm 10.29 \\
67.23 \pm 9.24\end{array}$ \\
\hline \multirow[t]{2}{*}{ Body Mass Index (BMI) } & $\begin{array}{l}\text { NEUROPATHY } \\
(\mathrm{n}=190)\end{array}$ & $\begin{array}{l}\text { OVERALL } \\
\text { MALE (145) } \\
\text { FEMALE (45) }\end{array}$ & $\begin{array}{l}26.35 \pm 3.32 \\
25.94 \pm 3.63 \\
28.12 \pm 4.27\end{array}$ \\
\hline & $\begin{array}{l}\text { NON-NEUROPATHY } \\
(n=190)\end{array}$ & $\begin{array}{l}\text { OVERALL } \\
\text { MALE (142) } \\
\text { FEMALE (48) }\end{array}$ & $\begin{array}{l}25.90 \pm 2.13 \\
25.40 \pm 3.66 \\
28.26 \pm 3.39\end{array}$ \\
\hline \multirow[t]{2}{*}{$\begin{array}{l}\text { Fasting Blood Sugar (FBS) in } \\
\mathrm{mg} / \mathrm{dL}\end{array}$} & $\begin{array}{l}\text { NEUROPATHY } \\
(\mathrm{n}=190\end{array}$ & $\begin{array}{l}\text { OVERALL } \\
\text { MALE (145) } \\
\text { FEMALE (45) }\end{array}$ & $\begin{array}{l}194.25 \pm 66.65 \\
193.96 \pm 70.34 \\
198.46 \pm 48.89\end{array}$ \\
\hline & $\begin{array}{l}\text { NON-NEUROPATHY } \\
(n=190)\end{array}$ & $\begin{array}{l}\text { OVERALL } \\
\text { MALE (142) } \\
\text { FEMALE (48) }\end{array}$ & $\begin{array}{l}158.43 \pm 48.43 \\
159.4 \pm 51.73 \\
152.26 \pm 32.17\end{array}$ \\
\hline $\begin{array}{l}\text { Post Prandial Blood Sugar } \\
\text { (PPBS) in } \mathrm{mg} / \mathrm{dL}\end{array}$ & $\begin{array}{l}\text { NEUROPATHY } \\
(\mathrm{n}=190)\end{array}$ & $\begin{array}{l}\text { OVERALL } \\
\text { MALE (145) }\end{array}$ & $\begin{array}{l}276.63 \pm 74.41 \\
278.47 \pm 78.34\end{array}$ \\
\hline
\end{tabular}

Diabetes peripheral neuropathy (DPN), Diabetic Foot Syndrome (DFS), Ankle Brachial Index (ABI), Ground Reaction Force (GRF), Body Mass Index (BMI), 


\begin{tabular}{|l|l|l|l|}
\hline & & FEMALE (45) & $285.43 \pm 55.18$ \\
\hline
\end{tabular}

191 Table2. Duration of diabetes and severity of neuropathy among DPN group.

\begin{tabular}{|c|c|c|}
\hline Parameters & \multicolumn{2}{|c|}{ Frequency (N \%) } \\
\hline \multirow{8}{*}{$\begin{array}{l}\text { Duration of } \\
\text { Diabetes with } \\
\text { Neuropathy }\end{array}$} & $1-5$ years & $56(29.47 \%)$ \\
\hline & 6-10 years & $63(33.15 \%)$ \\
\hline & $11-15$ years & $38(6.7 \%)$ \\
\hline & $16-20$ years & $19(20 \%)$ \\
\hline & 21-25 years & 07 (3.68\%) \\
\hline & $26-30$ years & $06(1.1 \%)$ \\
\hline & $>31$ years & $01(0.52 \%)$ \\
\hline & TOTAL & $190(100 \%)$ \\
\hline \multirow{4}{*}{$\begin{array}{l}\text { Grades of } \\
\text { Neuropathy } \\
\text { (Vibration } \\
\text { Pressure } \\
\text { Threshold } \\
\text { Testing) }\end{array}$} & MILD & $43(22.63 \%)$ \\
\hline & MODERTAE & $57(30 \%)$ \\
\hline & SEVERE & $90(47.36 \%)$ \\
\hline & TOTAL & $190(100 \%)$ \\
\hline
\end{tabular}

Table 3. Maximum Plantar Pressure Prediction: Parameter estimates using regression

\begin{tabular}{|c|c|c|c|c|c|c|}
\hline \multirow[b]{2}{*}{ Predictors } & \multirow[b]{2}{*}{ Estimates } & \multirow[b]{2}{*}{ t value } & \multirow[b]{2}{*}{$P$ value } & \multicolumn{2}{|c|}{ 95\% Confidence Interval } & \multirow{2}{*}{$\begin{array}{c}\text { Partial Eta } \\
\text { Squared }\end{array}$} \\
\hline & & & & Lower Bound & Upper Bound & \\
\hline \multicolumn{7}{|l|}{ GRADES NEUROPATHY } \\
\hline MILD & 168.758 & 16.806 & $<0.001$ & 149.035 & 188.482 & .337 \\
\hline MODERATE & 184.611 & 17.817 & $<0.001$ & 164.258 & 204.963 & .364 \\
\hline SEVERE & 186.004 & 19.656 & $<0.001$ & 167.416 & 204.591 & .410 \\
\hline NORMAL & & & & & & \\
\hline \multicolumn{7}{|l|}{ VARICOSITY } \\
\hline YES & 17.751 & 2.809 & 0.005 & 5.340 & 30.162 & .014 \\
\hline NO & & & & & & \\
\hline \multicolumn{7}{|l|}{ PLANTAR CUSHION } \\
\hline POOR & 19.724 & 4.356 & $<0.001$ & 10.830 & 28.617 & .033 \\
\hline FAIR & -3.239 & -0.683 & 0.495 & -12.558 & 6.079 & .001 \\
\hline GOOD & & . & & & & \\
\hline
\end{tabular}

Diabetes peripheral neuropathy (DPN), Diabetic Foot Syndrome (DFS), Ankle Brachial Index (ABI), Ground Reaction Force (GRF), Body Mass Index (BMI), 


\begin{tabular}{|l|r|r|r|r|r|r|}
\hline KMSA $\left({ }^{\circ}\right)$ & -1.106 & -2.574 & 0.010 & -1.950 & -0.262 & .012 \\
\hline NSS & 5.354 & 4.330 & 0.000 & 2.925 & 7.782 & .033 \\
\hline FOOTANGLE $\left({ }^{\circ}\right)$ & 1.049 & 2.427 & 0.016 & 0.200 & 1.898 & .010 \\
\hline KTOAA $\left(\% \mathbf{s}^{2}\right)$ & 0.032 & 2.844 & 0.005 & 0.010 & 0.054 & .014 \\
\hline KTOV $(\%)$ & 0.276 & 0.059 & $<0.001$ & 0.161 & 0.392 & .038 \\
\hline AHSV $(\%)$ & 0.735 & 0.176 & $<0.001$ & 0.390 & 1.081 & .031 \\
\hline
\end{tabular}

Diabetes peripheral neuropathy (DPN), Diabetic Foot Syndrome (DFS), Ankle Brachial Index (ABI), Ground Reaction Force (GRF), Body Mass Index (BMI), *KTOV- knee toe-off velocity, *AHSV- ankle heel strike velocity.

Adjusted $\mathrm{R}^{2}$ of the final model which included the predictors in table 1 was $90.8 \%$ The linear prediction equation obtained using regression analysis is

Predicted Maximum Plantar Pressure= 504.14 + 186 Severe Neuropathy +184.61 Moderate Neuropathy + 168.76 Mild Neuropathy + 17.75 Varicosity+ 19.72 Poor plantar cushion-3.24 Fair plantar fat pad - 1.11 KMSA + 5.36 NSS + 1.05 Foot angle+0.03 KTOAA+0.28 KTOV+0.74AHSV

Here, the predictors such as, Severe Neuropathy, Moderate Neuropathy, Mild Neuropathy, Varicosity, Poor plantar cushion and Fair plantar cushion are indicator variables (they take value 1 for presence and 0 for absence)

(1) 213 


\section{DISCUSSION:}

216

217

218

219

220

221

222

223

224

225

226

227

228

229

230

231

232

233

234

235

236

237

The clinical evaluation and clinical assessment of diabetic foot could help to reduce the risk of future foot complications. The present study highlights that many clinical parameters interact with each other alter the plantar pressure significantly, further damaging the foot and increasing the risk of ulceration among diabetes mellitus. The strength of our study lies in the number of variables assessed and to the best of our knowledge none of the published studies have reported so many variables in a single study with a regression euqation.

In the present study, we found a regression model which included kinematics and spatiotemporal parameters gait parameters in addition to neuropathy, vascular, musculoskeletal and other clinical findings against the Maximum Plantar pressure. The results from Table 3 suggested that variables like grades of neuropathy, presence of varicosity, plantar fat pat thickness, knee mid stance angle (KMSA, angle at knee joint during the midstance phase of gait cyle), Neuropathy Symptoms Score (NSS), Foot angle (degree of toe-out or line of progression ), Knee toe-off acceleration (KTOA, acceleartion at knee joint during toe-off phase of gait cycle), Knee toe-off velocity(KTOV,velocity at knee joint during toe-off phase of gait cycle) and ankle heel strike velocity (AHSV, velocity at ankle joint during heel strike phase of gait cycle) are significant predictors of maximum plantar pressure. The coefficient of determination $\left(\mathrm{R}^{2}\right)$ for the model is 0.908 which suggests that $90.8 \%$ of variability in maximum pressure is explained by the predictors in the model. The overall $F$ value for model adequacy was observed to be $F(12,568)=469.45$, with a corresponding singnficant $p$ value $<0.05$.Table3 also

Diabetes peripheral neuropathy (DPN), Diabetic Foot Syndrome (DFS), Ankle Brachial Index (ABI), Ground Reaction Force (GRF), Body Mass Index (BMI), 
provides the parameter estimates based on which the linear prediction equation has been developed. The equation could be explained as below:

240

The equation suggested that grades of neuropathy had a significant impact on maximum plantar pressure. The regression analysis showed that effect size (partial eta square) increased with an increase in the grade of neuropathy. It should also be noted that severe neuropathy could increase the maximum plantar pressure by $186 \mathrm{kPa}$ (Table 3) as compared to subjects without neuropathy. Similarly, the poor plantar fat pad in the feet could lead to increase of maximum plantar pressure by $19.72 \mathrm{kPa}$ whereas a fair fat pad could decrease it by 3.23 units as compared to good fat pad thickness. In the present analysis, variables like knee as well as ankle velocity and acceleration contributed significantly to the prediction model. It is observed that elevated values of knee toe-off velocity, acceleration, and ankle heel strike velocity lead to an increase in the maximum plantar pressure whereas increase in knee midstance angle decreases maximum plantar pressure. In other words, the greater the knee extension, the lower would be the maximum plantar pressure. In the present study, we find that participants with diabetes peripheral neuropathy had higher knee flexion angle at mid-stance. Findings from the present study supports that various clinical parameters could be responsible for increased maximum pressure which could increase the chances of future foot ulceration among neuropathy group. The model has highlighted the significance of varicosity in the lower limb suggesting that peripheral pooling of blood (collection of blood in the lower limb and reduced blood flow from extremity to the heart) in the feet could increase the maximum plantar pressure by $17.75 \mathrm{kPa}$ as compared to subjects without varicosity. The results of the present study are similar to the regression model proposed in the previous literature. However, it should be carefully understood that these models could be affected by choice of variables, the 
population characteristics, choice and the number of independent variables. To the best of our knowledge, this could be the first study to propose the Diabetic Foot prediction model with more than 150 variables among the Indian population. Ahroni et al. showed that high plantar pressure in diabetic population could be predicted with clinical parameters like body mass, insulin use, Caucasian race, gender (male), callus and diabetes duration [13]. In the present study, body mass was not retained in our clinical model. Cavanaugh et al. also suggested that body mass was a poor predictor [14]. However, results on gender and diabetes duration were in consensus with the previous finding and suggested that female gender showed decreased max. pressure by $19.18 \mathrm{kPa}$ at initial analysis but not retained in the final model. The results of the present study is also supported by findings of the study done by Barn et al. which reported that clinical factor like gender, body mass, diabetes duration, $\mathrm{HbA1c}$, VPT, foot ray (biomechanical axis of the ankle joint), foot deformity, ankle range of motion and callus were significant predictors of peak pressure among 167participants [15]. Similar findings were also reported by Fawzi et al. in the Egyptian population [16]. Few studies with regression analysis on gait kinetics and kinematics have been reported previosly. For instance, the study done by Wrobel et al. suggested that in the multivariate analysis for gait parameters age, ankle joint mobility, and callus were retained in the model with $17 \%$ variance for peak plantar pressure [17]. In the stepwise method, age showed $8.23 \%$ variance; ankle joint mobility showed 3.4\% and callus showed $1.4 \%$ variance. On the other hand, a study done by Guldemond et al. suggested callus and toe deformities as relevant predictors of peak forefoot pressure with $26 \%$ of the variance [18] .Similarly, the study done by Barn et al. suggested that Charcot foot showed the highest predictor value for peak pressure (Beta coefficient $=0.504$ ) [15] . In the forefoot, prominent metatarsal head showed the highest contribution of $31 \%$ followed by claw toes. In the present study, we excluded Charcot foot Diabetes peripheral neuropathy (DPN), Diabetic Foot Syndrome (DFS), Ankle Brachial Index (ABI), Ground Reaction Force (GRF), Body Mass Index (BMI), 
however the findings were consistent with prominent metatarsal heads and clawing of toes at early stage of analysis. However these factors were not retained in the final model. The study done by Fawzy et al. [16] suggested that multivariate logistical regression analysis for ulceration risk was statistically significant for duration of diabetes (odds ratio $[\mathrm{OR}]=0.8$ ), smoking $(\mathrm{OR}=9.7)$, foot deformity $(\mathrm{OR}=8.7)$, MNDS $(\mathrm{OR}=1.5), 2$-h postprandial plasma glucose $(2 \mathrm{~h}$ $\mathrm{PPG})(\mathrm{OR}=0.9)$, glycated hemoglobin $(\mathrm{HbA1c})(\mathrm{OR}=2.1)$, forefoot peak plantar pressure (FFPP,OR $=1.0$ ), and forefoot peak plantar pressure gradient (FFPPG , $\mathrm{OR}=1.0$ ).The study done by Al-Rubeaan et al. reported that the risk factors like Charcot's joints, peripheral vascular disease, neuropathy, diabetes duration $\geq 10$ years, insulin use, retinopathy, nephropathy, age $\geq 45$ years, cerebral vascular disease, poor glycemic control, coronary artery disease, smoking, and hypertension was strongly associated with diabetic foot complications [19]. Also the present clinical model is in consensus with the previous findings with the addition of few more variables. For e.g., the thickness of plantar fascia or plantar fat pad could be an important clinical factor for prediction of peak pressure and future risk of ulceration among participants with diabetes [20].The mechanical properties of plantar soft tissue can be used to improve the predictability of diabetic foot ulcers in moderate/ high-risk patients [21].

From the present study, it is evident that the given regression model for diabetic foot prediction could be an important tool in day to day clinical evaluation for predicting the maximum plantar pressure and minimizing future foot complications. Therefore future studies should be done to test the model. Studies have reported a threshold values for peak plantar pressure. The study from Armstrong et al. reported that a pressure of $60 \mathrm{~N} / \mathrm{cm}^{2}$ is the upper threshold for development of an ulcer in diabetes mellitus [22]. The study also reported a cut-off 
317

point of $335 \mathrm{kPa}$ (peak plantar pressure) was considered as a risk, for ulceration at forefoot [22]. However, findings from recent studies have been contradictory. The study done by Bus et al. suggested that plantar pressure threshold should not be considered as the suitable method for detecting the risk of foot ulceration in participants with diabetes [23]. Nevertheless, the higher plantar pressure in the presence of sensory and motor neuropathy could be a potential risk for foot ulceration [24]. Thus, higher plantar pressure could be significantly associated with deformities and soft tissue changes in the foot. Findings from the present study could help to strengthen the importance of plantar pressure threshold values with future experimental studies.

Future Scope: The use of the given model could be useful and easier with advanced biomechanical labs with motion analysis. However it could also be extended to communities with 2D video analysis through Smartphone's and freely available software's for video analysis to calculate joint angle, velocity and acceleration. For instance the angular velocity could be obtained by rate change of angular displacement (radians). Similarly, angular acceleration could be obtained by the rate change of angular velocity. The study could be useful to carry out plantar pressure analysis in a clinical population even in the absence of advanced 3D motion analysis system using clinical parameters. However, future studies need to work out more on the proposed model which could be more user friendly at clinical and community levels.

CONCLUSION: The suggested model was found be strongly significant in determining the maximum plantar pressure which could be associated with risk of future foot complications. The study highlighted the most important clinical parameters while assessing a diabetic foot with neuropathy. Based on the findings remedies to control plantar pressure could be suggested and rehabilitation protocol Diabetes peripheral neuropathy (DPN), Diabetic Foot Syndrome (DFS), Ankle Brachial Index (ABI), Ground Reaction Force (GRF), Body Mass Index (BMI), 
could be prepared. We believe that the given model is in its primary stage and future modifications could be required to make it more efficient and user friendly for routine clinical practice.

Acknowledgement: I am grateful and would like to acknowledge World Diabetes

care and Research, MAHE for all the support provided towards recruitment of the

\section{REFERENCES:}

1. Sawacha Z, Cristoferi G, Guarneri G, Corazza S, Donà G, Denti P, Facchinetti A, Avogaro A, Cobelli C. Characterizing multisegment foot kinematics during gait in diabetic foot patients. Journal of NeuroEngineering and Rehabilitation. 2009 Dec;6(1):37.

2. Papanas N, Maltezos E. The diabetic foot: a global threat and a huge challenge for Greece. Hippokratia. 2009 Oct;13(4):199.

3. Tuttolomondo A, Maida C, Pinto A. Diabetic foot syndrome: Immune-inflammatory features as possible cardiovascular markers in diabetes. World journal of orthopedics. 2015 Jan 18;6(1):62.

4. Dyck PJ, Herrmann DN, Staff NP, Dyck PJ. Assessing decreased sensation and increased sensory phenomena in diabetic polyneuropathies. Diabetes. 2013 Nov 1;62(11):3677-86.

5. Kwon OY, Tuttle LJ, Johnson JE, Mueller MJ. Muscle imbalance and reduced ankle joint motion in people with hammer toe deformity. Clinical Biomechanics. 2009 Oct 1;24(8):670-5.

6. Verrotti, A., Prezioso, G., Scattoni, R. and Chiarelli, F. Autonomic neuropathy in diabetes mellitus. Frontiers in endocrinology 2014;(5):205.

7. Petrofsky J, Berk L, Al-Nakhli H. The influence of autonomic dysfunction associated with aging and type 2 diabetes on daily life activities. Experimental diabetes research. 2012 Apr 9;2012.

8. Bacarin TA, Sacco IC, Hennig EM. Plantar pressure distribution patterns during gait in diabetic neuropathy patients with a history of foot ulcers. Clinics. 2009 Feb;64(2):113-20.

9. Ledoux WR, Shofer JB, Cowley MS, Ahroni JH, Cohen V, Boyko EJ. Diabetic foot ulcer incidence in relation to plantar pressure magnitude and measurement location. Journal of Diabetes and its Complications. 2013 Nov 1;27(6):621-6.

10. Ghosal, S., Stephens, J. and Mukherjee, A., 2012. Quantitative vibration perception threshold in assessing diabetic neuropathy: Is the cut-off value lower for Indian Subjects?[Q-VADIS Study]. Diabetes \& Metabolic Syndrome: Clinical Research \& Reviews, 6(2), pp.85-89.

11. Feng, Y., Schlösser, F.J. and Sumpio, B.E., 2009. The Semmes Weinstein monofilament examination as a screening tool for diabetic peripheral neuropathy. Journal of Vascular Surgery, 50(3), pp.675-682.

12. Kaur, J. and Batra, A.P.S., 2016. Vibration perception threshold as a measure of distal symmetrical neuropathy in type 2 diabetes. International Journal of Contemporary Medical Research, 3, pp.1839-1841.

13. Ahroni JH, Boyko EJ, Forsberg RC. Clinical correlates of plantar pressure among diabetic veterans. Diabetes care. 1999 Jun 1;22(6):965-72.

14. Cavanagh PR, Sims DS, Sanders LJ. Body mass is a poor predictor of peak plantar pressure in diabetic men. Diabetes care. 1991 Aug 1;14(8):750-5.

Diabetes peripheral neuropathy (DPN), Diabetic Foot Syndrome (DFS), Ankle Brachial Index (ABI), Ground Reaction Force (GRF), Body Mass Index (BMI), 
18. Guldemond NA, Leffers P, Walenkamp GH, Schaper NC, Sanders AP, Nieman FH, van Rhijn LW. Prediction of peak pressure from clinical and radiological measurements in patients with diabetes. BMC endocrine disorders. 2008 Dec;8(1):16.

19. Al-Rubeaan K, Al Derwish M, Ouizi S, Youssef AM, Subhani SN, Ibrahim HM, Alamri BN. Diabetic foot complications and their risk factors from a large retrospective cohort study. PloS one. 2015 May 6;10(5):e0124446.

20. Kumar CG, Rajagopal KV, Hande HM, Maiya AG, Mayya SS. Intrinsic foot muscle and plantar tissue changes in type 2 diabetes mellitus. Journal of diabetes. 2015 Nov 1;7(6):850-7.

21. Naemi R, Chatzistergos P, Suresh S, Sundar L, Chockalingam N, Ramachandran A. Can plantar soft tissue mechanics enhance prognosis of diabetic foot ulcer?. diabetes research and clinical practice. 2017 Apr 1;126:182-91.

22. Armstrong DG, Lavery LA, Vela SA, Quebedeaux TL, Fleischli JG. Choosing a practical screening instrument to identify patients at risk for diabetic foot ulceration. Archives of internal medicine. 1998 Feb 9;158(3):289-92.

23. Bus SA. Innovations in plantar pressure and foot temperature measurements in diabetes. Diabetes/metabolism research and reviews. 2016 Jan 1;32(S1):221-6.

24. Hazari A, Maiya AG, Shivashankara KN, Monteiro MA, Kumar CS, Rao K, Kumar S, Maiya SS, Jadhav R. 3D biomechanical analysis of foot in diabetes with and without peripheral neuropathy-a pilot study. Research Journal of Pharmaceutical, Biological and Chemical Sciences. 2016;7(3):558-64. 
'Conflict of interest: none' 